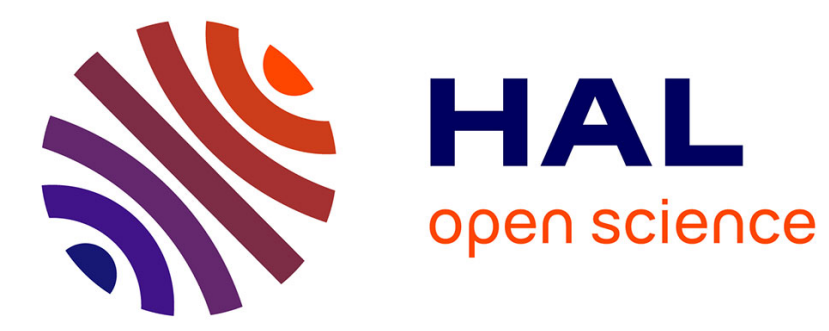

\title{
Crack stability in rate sensitive ceramics and their rate dependence
}

\author{
T. Nojima
}

\section{To cite this version:}

T. Nojima. Crack stability in rate sensitive ceramics and their rate dependence. Journal de Physique IV Proceedings, 1994, 04 (C8), pp.C8-689-C8-694. 10.1051/jp4:19948105 . jpa-00253346

\section{HAL Id: jpa-00253346 https://hal.science/jpa-00253346}

Submitted on 1 Jan 1994

HAL is a multi-disciplinary open access archive for the deposit and dissemination of scientific research documents, whether they are published or not. The documents may come from teaching and research institutions in France or abroad, or from public or private research centers.
L'archive ouverte pluridisciplinaire HAL, est destinée au dépôt et à la diffusion de documents scientifiques de niveau recherche, publiés ou non, émanant des établissements d'enseignement et de recherche français ou étrangers, des laboratoires publics ou privés. 
JOURNAL DE PHYSIQUE IV

Colloque C8, supplément au Journal de Physique III, Volume 4, septembre 1994

\title{
Crack stability in rate sensitive ceramics and their rate dependence
}

\author{
T. Nojima
}

Dept. of Aeronautical Engineering, Kyoto University, Sakyo-ku, Kyoto 606, Japan

\begin{abstract}
The crack stability condition in rate sensitive brittle materials is analysed. The analysis shows that a crack begins to extend before a maximum load $\left(P_{\text {max }}\right)$ appears in fracture toughness $\left(K_{T}\right)$ tests and the extension depends on the $n$-value in the $\dot{a}^{\propto} K_{I}^{n}$ relation. This suggests that the evaluation of a true $K_{T}$-value is quite difficult. An analytical method for using the $K_{T ;}$ -value (the initial stress intensity evaluated by the $P_{\text {max }}$ and initial crack length) is proposed. Experimental data for an alumina ceramic and a glass are analysed by the method. The $K_{I i}-T_{f}$ relation of the glass consists of 4 regions over the range $10^{-5} \mathrm{~s}<T_{f}<10^{7} \mathrm{~s}$ where $T_{f}$ is the time to failure.
\end{abstract}

\section{INTRODUCTION}

It has been experimentally clarified to a great extent that the fracture toughness value $K_{I}$ or strength $\sigma_{0}$ of glasses and ceramics shows a considerable rate effect. In some oxide ceramics such as glasses and alumina ceramics [1, 2], because crack extension is affected by the stress corrosion caused by water in the environment, a trimodal curve is frequently obtained for $K_{T}$ - $\dot{a}$ relation ( $\dot{a}$; the crack velocity); the rate dependence generally consists of 3 regions (Region I UII). In order to interpret these different rate dependences, it is necessary not only to accumulate further precise data but to analyze the data by utilizing fracture mechanics including rate effects.

Nevertheless, the crack stability condition including the rate effect has been left undiscussed and analytical methods for the data on cracked specimens are not available. Moreover, as mentioned above, although it has been well known that the $K_{I}-\dot{\alpha}$ relations for oxide ceramics, for example, are not simple, no careful data analysis has been done in determining $K_{I}-V_{0}$ (or $\sigma_{0}-V_{0}, V_{0}$; the testing speed) relations.

In the first part of the present paper, the crack-stability including the rate effect is discussed and a pseudo-stable phenomenon is explained. A new method to analyze $K_{I}$-data for different initial crack length and specimen geometry is presented. In the second part, some experimental data and analyzed results on a glass and an alumina ceramic are shown. 


\section{STABILTTY CONDITION OF SPECTMENS WITH A CRACK}

Consider the fracture toughness tests of brittle materials in a conventional testing machine. By assuming a quasi-static crack extension (kinetic energy=0) during the tests, the cross-head displacement $\delta$-load $P$ relation is expressed by $\delta=P\left(\lambda+\Lambda^{*}\right)$, where $\Lambda^{*}$ is the compliance of the testing machine and $\lambda(A)$ is the compliance of the specimen ( $A$; cracked area). Putting the energy release rate $G=\left(P^{2} / 2\right)(\mathrm{d} \lambda / \mathrm{dA})$ equal to the crack resistance force $R$, $\delta$ and $P$ can be expressed by

$$
\delta=\sqrt{2 B R /(\mathrm{d} \lambda / \mathrm{d} a)} \cdot\left\{\lambda(A)+\Lambda^{*}\right\}, \quad P=\sqrt{2 B R /(\mathrm{d} \lambda / \mathrm{d} a)},
$$

where $B$ is the thickness of the specimen and $a$ is the crack length $(A=B \alpha)$. By taking logarithms of both sides in Eq.(1) and differentiating with respect to the time $t$, the following Eq. (2) is obtained;

$$
\begin{aligned}
& 2(\dot{\delta} / \delta)=\left(\gamma^{*}-\eta^{*}\right) \dot{a}+\gamma^{* *} \ddot{a}, \quad \gamma^{*}=(\partial R / \partial \alpha)_{\dot{a}} / R, \quad \gamma^{* *}=(\partial R / \partial \dot{\alpha})_{a} / R, \\
& \eta^{*}(a)=\left(\mathrm{d}^{2} \lambda / \mathrm{d} a^{2}\right) /(\mathrm{d} \lambda / \mathrm{d} a)-2(\mathrm{~d} \lambda / \mathrm{d} a) /\left(\lambda+\Lambda^{*}\right) \text {. }
\end{aligned}
$$

The parameter $\gamma^{*}$ and $\gamma^{* *}$ are related to the material properties of the test samples, while $\eta^{*}$ is given by the testing conditions. In crack-rate insensitive materials $\left(\gamma^{* * *}=0\right)$, Eq. (2) becomes $2(\dot{\delta} / \delta)=\left(\gamma^{*}-\eta^{*}\right) \dot{\alpha}$. The following equation, which gives $\dot{\alpha}>0$, when $\delta>0$, becomes the stability condition for crack-rate insensitive materials (Gurney and Hunt [3]);

$$
\gamma^{*}-\eta^{*}>0 \text {. }
$$

In perfectly brittle materials $\left(\gamma^{*}=0\right), n^{*}<0$ gives the condition for a stable crack extension. Fig.1 shows a numerically calculated example for $P-\delta$ curves in the materials where $\gamma^{*}=0$ and $\gamma^{* *=0}$, for a single edge cracked 3 point bending specimen (SEC3PB, $x_{0}=\alpha_{0} / W, a_{0}$; initial crack length and $W$; specimen height, span length $S=4 W)$, where $\widetilde{P}=R / \sqrt{R_{0} E^{3} B^{2} W}, \widetilde{\delta}=\delta / \sqrt{R_{0} W / E^{3}}, \quad E^{\prime}=E /\left(1-v^{2}\right)$ with $E$ and $v$ the Young's modulus and Poisson's ratio of the specimen, respectively. The points plotted in the figure are for equiliblium for a given $x=\alpha / \mathrm{W}$. The top point of each curve is for $x=x_{0}$. According to the criterion by Gurney and Hunt, the curves which show $\mathrm{d} \delta>0$ at $x=x_{0}$ correspond to stable crack extension (symbol s), while the curves for d $d<0$ at $x=x_{0}$ correspond to unstable crack extension (symbol u). By their criterion, which neglects any rate effect, it has been interpreted that the load shows a sudden drop from its maximum value.

The value of $\gamma^{* *}$ is reported to be positive. Now we consider a test in which cross-head displacement is suddenly stopped during crack extension. Putting $\delta=0$ in Eq. (2), Eq (2) becomes $\left(\gamma^{*}-\eta^{*}\right) \dot{\alpha}+\gamma^{*} * \ddot{\alpha}=0$. From this equation, we easily understand that $\dot{a}$ accelerates with $t$ when $\gamma^{*}-n^{*}<0$, while $\dot{a}$ gets smaller with $t$ when $\gamma^{*}-n^{*}>0$. If we define that a crack is unstable when it accelerates itself without any external work $(\dot{\delta}=0)$, the stability condition for rate sensitive materials is also expressed by Eq. (3), i.e., $\gamma^{*}-\eta^{*}>0$.

In almost all the $K_{I}$ tests, the critical $K_{I}$ value has been evaluated by using the $P_{\text {max }}$ in the tests. Therefore it can be reasonably accepted that the crack is stable until a maximum appears in the $P-\delta$ curve. Thus, the $P$ max condition is deduced hereafter. By differentiating $P$ in Eq. (1) with respect to $t$, the following Eq. (4) is obtained;

$$
2(\dot{P} / P)=\left\{\gamma^{*}-\left(\mathrm{d} \lambda^{2} / \mathrm{d} \alpha^{2}\right) /(\mathrm{d} \lambda / \mathrm{d} \alpha)\right\} \dot{\alpha}+\gamma * * \ddot{\alpha}
$$

$P_{\max }$ is given by $\dot{P}=0$. In the crack rate insensitive material $\left(\gamma^{* * 0}\right)$, $P_{\max }$ will appear when the following Eq. (5) is satisfied;

$$
\gamma^{*}(a)-\left(\mathrm{d} \lambda^{2} / \mathrm{d} a^{2}\right) /(\mathrm{d} \lambda / \mathrm{d} a)=0
$$

Since $\left(\mathrm{d} \lambda^{2} / \mathrm{d} \alpha^{2}\right) /(\mathrm{d} \lambda / \mathrm{d} \alpha)>0$ in conventional specimens, $P_{\max }$ appears at $a=\alpha_{0}$ in perfectly brittle materials.

On the other hand, in the materials with positive rate effect $\left(\gamma^{* *}>0\right)$, the following equation holds when $P_{\max }$ appears;

$$
\gamma^{*}(a)-\left(\mathrm{d}^{2} \lambda / \mathrm{d} a^{2}\right) /(\mathrm{d} \lambda / \mathrm{d} a)+\gamma *(\ddot{a} / \dot{a})=0
$$


From Eq. (2), $\ddot{a}$ becomes positive when the instability condition $\gamma^{*}-\eta *<0$ is satisfied, since $\dot{\delta} / \delta>0$ (from testing condition), $\gamma * *>0$ and $\dot{a}>0$. By putting $\ddot{a}>0$ in Eq. (5), the third term becomes positive. The crack length which gives $P_{\max }$ in crack-rate insensitive materials is denoted as $a_{\max }^{0}$ (solution value of Eq.(5)), while the crack length in rate sensitive materials is denoted as a fax (solution value of Eq.(6)). The comparison between Eqs.(5) and (6) indicates that $a_{m a x}^{*}$ is larger than $a_{m a x}^{0}$ This can be more easily understood by the schematic illustration in Fig.2, where a method to determine these values is shown. Since the value of $\gamma^{* *}(\ddot{\alpha} / \dot{a})=h(\alpha)$ in $\mathrm{Eq} .(6)$ is positive, $a_{\max }^{*} a_{\max }^{0}$ obviously holds as far $\ddot{\alpha}>0$. When the specimen geometry, the testing condition and also the rate dependence of $R$ are given, $\dot{a}$ and $\ddot{a}$ in Eq. (6) will be calculated as a function of $a$ and $a_{m a x}^{*}$ can be determined (see next section).

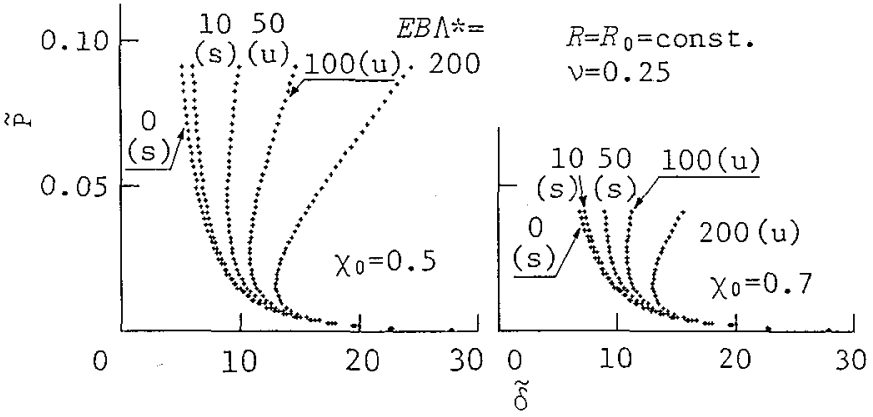

Fig.l $P-\delta$ curves for perfectly brittle, crack-rate insensitive materials $\left(\gamma^{*}=0, \gamma^{* *}=0\right)$

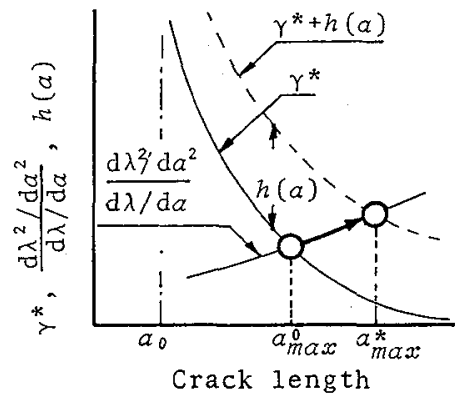

Fig. 2 Schematic illustration for determinig $a_{m a x}^{*}$ and $a_{\max }$

\section{NUMERICAL CALCULATIONS FOR $P-\delta$ RELATIONS AND CRACK EXTENSION $\triangle x$ BEFORE $P_{\text {max }}$}

The $\dot{a}-K_{I}$ relations in glasses and ceramics are often expressed by a power relation as $\dot{\alpha} \propto\left(K_{T}\right)^{n}$ ( $n=$ constant). By using $G=R=K_{T}^{2} / E^{\prime}$, it follows that $R \propto \dot{\alpha}^{2 / n}$. Therefore, in the following calculations, Eq.(7) is adopted as constitutive relations for these materials.

$$
R=R_{1}(a) \cdot\left(\dot{a} / \dot{a}^{*}\right)^{2 / n},\left(\dot{a} / \dot{a}^{*}\right)=\left(K_{I} / K_{I}^{*}\right)^{n} \quad\left(\dot{a}^{*} \text { and } K_{I}^{*} \text { are constants }\right) .
$$

In constant velocity tests in a conventional testing machine, i.e., $\delta=V_{0} t$, by substituting $R$ in Eq. (7) into Eq. (1), and also putting $\delta_{0}(a)=\sqrt{2 B R(a) /(\mathrm{d} \lambda / \mathrm{d} a)}$. $\left\{\lambda(\alpha)+\Lambda^{*}\right\}, \quad\left(\delta_{0}=\right.$ the $\delta$ for $\left.\gamma^{* *}=0\right)$, we find

$$
\delta^{2}=\left\{\delta_{0}(\alpha)\right\}^{2}\left(\dot{a} / \dot{a}^{*}\right)^{2 / n}=\left(V_{0} t\right)^{2} .
$$

By integrating Eq. (8), $P=\sqrt{2 \overline{B R} 1 /(\mathrm{d} \lambda / \mathrm{d} a)} \cdot\left(\dot{a} / \dot{a}^{*}\right) \mathrm{l} / n$

$$
P(a)=\sqrt{2 B R_{1}} /(\mathrm{d} \lambda / \mathrm{da})\left[v_{0}(1+n) / \dot{a}^{*}\right]^{\frac{1}{1+n}}\left\{\int_{a_{0}}^{a} \delta_{0}{ }^{n} \mathrm{~d} a\right\}^{\frac{1}{1+n}} / \delta_{0} \text {. }
$$

The $\delta$ is given by $\delta(\alpha)=P\left(\lambda+\Lambda^{*}\right)$. By taking the logarithm and defferentiating Eq. (9), it is found

$$
2 \frac{(d P / d t)}{P}=\left[\frac{\left(d R_{1} / d a\right)}{\bar{R}_{1}}-\frac{\left(d^{2} N d a^{2}\right)}{(d \lambda / d a)}+\left|\frac{2}{(1+n)} \frac{\delta_{0}{ }^{n}}{\int_{\infty}^{a} \delta_{0}{ }^{n} d a}-2 \frac{\left(d \delta_{0} / d a\right)}{\delta_{0}}\right|\right] \dot{a} .
$$

Since $2\left(\mathrm{~d} \delta_{0} / \mathrm{d} \alpha\right) / \delta_{0}=\gamma^{*}-\eta *$, the third term of the right hand side of Eq. (10) becomes positive, when $\gamma^{*}-\eta *<0$, and it corresponds to $\gamma^{* *}(\ddot{\alpha} / \dot{a})$ in Eq. (6). By the numerical calculations, it was also clarified that $\ddot{a}$ is positive at least up to $P_{\text {max }}$ even when $\gamma *-n *>0$.

Fig. 3 shows the numerically calcurated $P-\delta$ curves for $\operatorname{SEC} 3 P B\left(\gamma^{*}=0\right)$. Calculated $P-$ values are plotted at an interval of $\Delta x=0.01$. In the figure, it can be seen that a crack begins to extend before $P_{m a x}$. The phenomenon of a load increase when the crack continues to extend is named pseudo-stable phenomenon (PSP). The crack extention $(\Delta x)$ appears more remarkably as the $n$-value gets smaller or $E B \Lambda^{*}$ becomes larger. The $P_{\text {max }}$ value, which is commonly utilized to evaluate $K_{I}$ (or $K_{I c}$ ), also depends on the testing condition $E B \Lambda *$. The dependence comes to appear more remarkably as the $n$ value becomes smaller. As an important consequence, it is argued that attention should be paid in checking the crack extension before the $K_{I}$-value is calculated from the maximum load. In the above discussion on the crack stability in rate sensitive brittle materials, the stable/unstable condition was interpreted by 
considering whether a crack is accelerated or not when no external work is supplied. However it will be also natural to consider that a crack is stable up to the maximum load. From this point of view, it can be said that a crack in rate sensitive materials becomes more stable $\left(\Delta \alpha=a_{m a x}-a_{0}\right)$ than that of rate insensitive materials.

By putting $\dot{P}=0$ in Eq. (10), the critical crack length ( $x_{m}$ ax) at $P_{\text {max }}$ can be calculated numerically. The amount of the crack extension, $\Delta x=\Delta a / W$, is shown in Fig.4 ((A) and (B); for SEC3PB, (C); for single edge cracked tensile specimen, SECT). The figure shows two important facts; (1) the $\Delta x$-value is a function of $x_{0}$ and it decreases with $x_{0}$ and (2) the $\Delta x$-value depends on the specimen geometry and the value in SECT is larger than the one in SEC3PB. These facts suggest that in perfectly brittle materials, the values of $K_{I i}$ (the initial stress-intensity), as conventionally evaluated by utilizing $P_{m a x}$ and $a_{0}$ values, will inevitably increase with $x_{0}$ and the $K_{I i}$-value in SEC3PB will be larger than the one in SECT. The analyzed predictions have been clarified in a soda-lithe glass and an alumina ceramic by using these specimens [4].

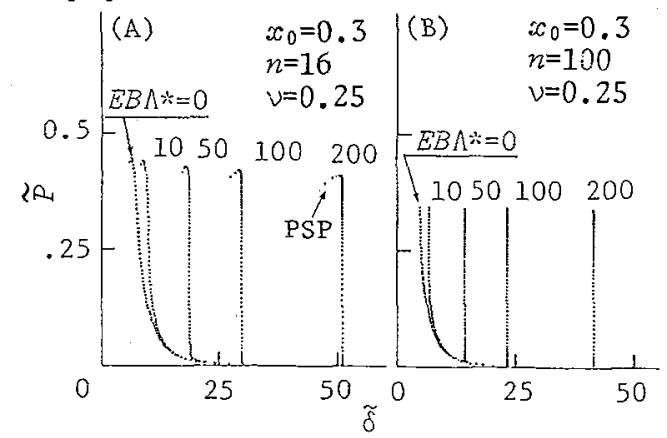

Fig. 3 Simulated $P-\delta$ relations for crack-rate sensitive materials showing pseudo-stable phenomenon (PSP) $\left(\gamma^{*}=0, \gamma^{* *}>0\right.$, SEC 3PB)

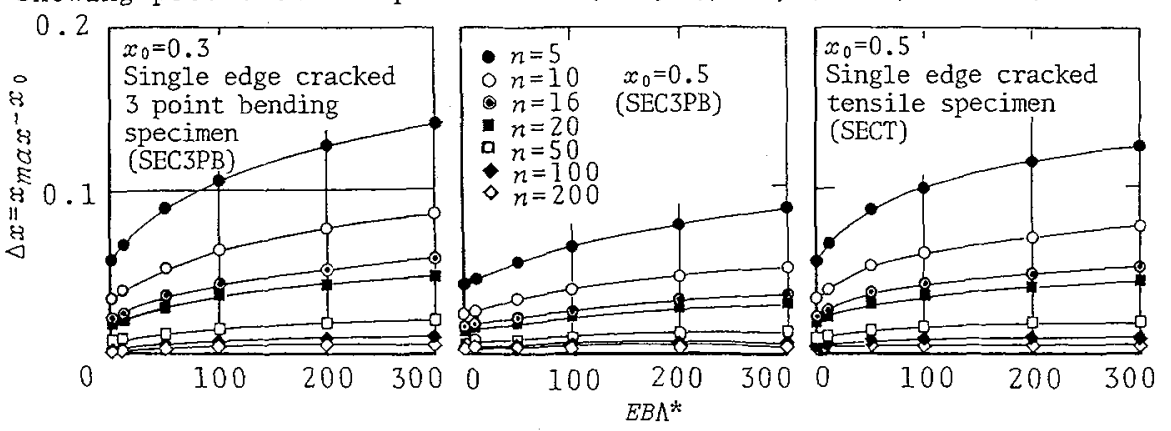

Fig. 4 Non-dimensional crack-extension before $P_{\text {max }}$ as a function of $E B \Lambda^{*}$

4. A NEW ANALYTICAL METHOD FOR EXPERIMENTAL DATA ON RATE SENSITIVE-BRITTLE MATERIALS WITH DIFFERENT INITIAL CRACK LENGTHS

Experimental data for delayed failuxe in ceramics have been analyzed by a simple formula presented by Evans [2]. The formula can not be applied directly to the data for large initial cracks $\left(x_{0}>0.1\right)[5]$. Thus a new method of data analysis for any crack length is presented in this section.

By expressing the non-dimensional compliance of the specimen as $\tilde{\lambda}=\lambda /\left(2 / E^{\prime} B\right)$ and substituting this relation into $K_{I}^{2} / E^{\prime}=\left\{P(t)^{2} / 2 B\right\}\{\mathrm{d} \lambda / \mathrm{d} \alpha\}, K_{I}$ value becomes $K_{I}=P(t) /(B \sqrt{W}) \cdot \sqrt{\mathrm{d} \lambda / \mathrm{d} x}$. By substituting the $K_{I}$ into Eq. (7), the following relation is obtained;

$$
\left(\dot{\alpha} / \dot{a}^{*}\right)=\left\{P(t) /\left(B \sqrt{W} K_{I}^{*}\right)\right\}^{n}\{\mathrm{~d} \tilde{\lambda} / \mathrm{d} x\}^{n / 2}
$$

By putting $\dot{\alpha}=W \dot{x}$ and integrating $\mathrm{Eq} \cdot(11)$, Eq. (11) becomes Eq. (12).

$$
\int_{x_{0}}^{x}(d \tilde{\lambda} / d x)^{-n / 2} d x=\left(\dot{a}^{*} / W\right) /\left(B \sqrt{W} \cdot K_{I}^{*}\right)^{n} \cdot \int_{0}^{t}\{P(t)\}^{n} d t .
$$


By putting $P=\alpha t$ ( $\alpha=$ constant) in constant loading rate (CLR) tests (conventional test), the integration of the right hand side in Eq. (12) becomes $\left(P_{m a x}\right)^{n \cdot T}$ 㧘 $/(n+1)$ ( $T_{f}^{*}$; the time to the maximum load, $P_{\max }=\alpha T_{f}^{*}$ ), while in constant load (CL) test, it becomes $P_{0} n^{n} T_{f}$. The time to failure in this test is denoted as $T_{f}$ :

By definig that "a specimen fractures when $x=1$ ", Eq. (12) becomes

$$
\begin{aligned}
& \int_{x_{0}}^{1}(\mathrm{~d} \tilde{\lambda} / \mathrm{d} x)^{(-n / 2)} \mathrm{d} x=\left(\dot{\alpha}^{*} / W\right)\left(\widetilde{P}_{\max }\right)^{n} \cdot\left\{T_{f}^{*} /(n+1)\right\} \quad \text { for CLR tests, } \\
& \int_{x_{0}}^{1}(\mathrm{~d} \widetilde{\lambda} / \mathrm{d} x)^{(-n / 2)} \mathrm{d} x=\left(\dot{a}^{*} / W\right)\left(\widetilde{P}_{0}\right)^{n} \cdot T_{f} \quad \text { for CL tests. }
\end{aligned}
$$

The initial stress intensity $K_{I i}$ is expressed by $K_{I i}=P_{c} /(B \sqrt{W}) \cdot \sqrt{x_{0}} \dot{F}\left(x_{0}\right)$, where $F$ is the geometry factor of the specimen, and $P_{C}$ is $P_{\max }$ or $P_{0}$ in the CLR and CL tests, respectively. By introducing this equation into Eq.(13), and also putting $(\mathrm{d} \lambda / \mathrm{d} x)=x F^{2}$ (see Appendix), the following equation is obtained;

$$
\left(W / \dot{a}^{*}\right) \int_{x_{0}}^{1}\left\{\sqrt{x_{0}} E\left(x_{0}\right) / \sqrt{x} F(x)\right\}^{n} \mathrm{~d} x=\left(K_{I i} / K_{I}^{*}\right)^{n} \hat{T}_{f} .
$$

where $\hat{T}_{f}$ is $T+(n+1)$ or $T_{f}$ in the CRL and CL tests, respectively.

Eq. (14) indicates that the $K_{I i}$-values for different initial crack lengths $\left(x_{0}\right)$, the specimen geometry $(F)$ and the size (W) can be inclusively expressed by the following new parameter (non-dimensional normalized time to failure)

$$
\left(\widetilde{T_{f}}\right)_{N}=\left\{\hat{T}_{f} /\left(W / \dot{\alpha}^{*}\right)\right\} I^{-1}, \quad I=\int_{x_{0}}^{1}\left\{\frac{\sqrt{x} \cdot \cdot F\left(x_{0}\right)}{\sqrt{x} \cdot F(x)}\right\}^{n} \mathrm{~d} x
$$

In the above analysis, it was assumed that the $K_{T}-\dot{a}$ relation is given by a single power relation. However the $K_{I} \dot{\alpha}$ relations in oxide ceramics of ten show trimodal curves [1,2] (Fig.5). The figure shows 4 regions including Region 0 for the threshold of $K_{T}(K)$. A sharp gap in $K_{T}$ appears in Region II (the crack velocity of Region II is about $10^{-3} \mathrm{~m} / \mathrm{s}$ for an alumina and about $10^{-3.5} \mathrm{~m} / \mathrm{s}$ for a soda-lime glass in an ambient environment).

$K_{I i-T_{f}}$ relations were simulated by using the $K_{T}-\dot{a}$ relation in Fig.5 $\left(n_{0}=\infty, n_{1}=20\right.$, $n_{2}=0$ and $n_{3}=20$ ) [5]. The simulated results shown in Fig.6 clarify that the $K_{I i}-T_{f}$ relations depend on the $x_{0}$-value, and the sharp gap appears as a gentle curve in the $K_{I i}{ }^{T} f$ relation. However, if we adopt the normalized time to failure $\left(\widetilde{T}_{f}\right)_{N}$, it is seen in Fig.7 that the $K_{I i}$-values can be expressed by a unique function of ( $\left.T_{f}\right)_{N}$. When the $n$-values are different in Region I and III, the experimental data for delayed failure can be analyzed by using their respective values [5].

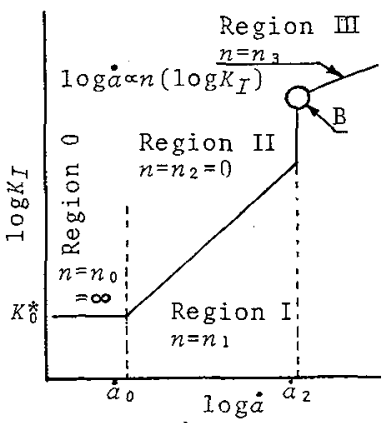

Fig. 5 Schematicillustration of $K_{I}-\dot{a}$ relation of oxide ceramics showing 4 regions

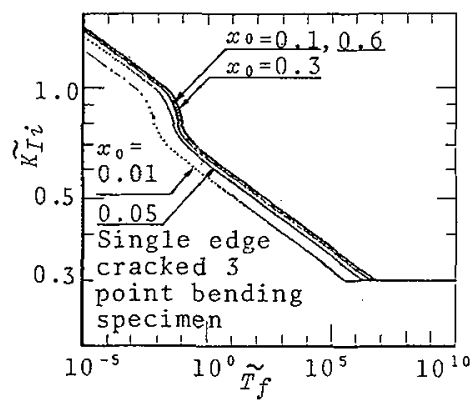

Fig. $6 \log K_{I i}-\log T_{f}$ relations for various kinds of initial crack length (simulated, SEC 3PB)

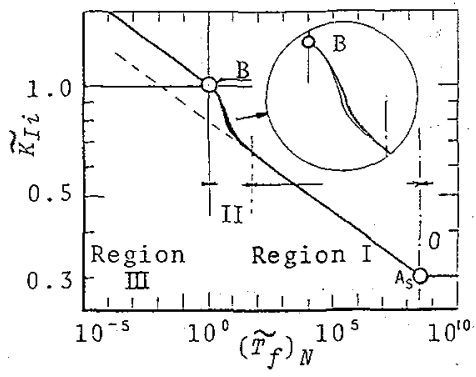

Fig. $7 \log K_{I i}-\log \left(\widetilde{T_{f}}\right)_{N}$ relation ( (Tf) normalized time to failure, simulated, SEC 3PB)

\section{EXPERIMENTAL DATA AND THEIR ANALYSIS}

Because a crack begins to extend before $P_{\max }$ in rate-sensitive materials, it is quite difficult to evaluate true $K_{I}$-values. Thus the experimental data will be analyzed by the method for $K_{I i}$ in Section 3 .

Fracture toughness tests of an alumina ceramic $(96 \%$ alumina, $B=1 \mathrm{~mm}, W=8 \mathrm{~mm}$, Kyocera) and a soda-lime glass $(B=1.8 \mathrm{~mm}, W=19 \mathrm{~mm})$ were carried out on about 250 SEC3PB specimens at $V_{0}=0.005-5 \mathrm{~mm} / \mathrm{min}$, and at two kinds of slower loading rate by using a coil spring installed in loading bar. The obtained $K_{I i}-T_{\vec{f}}^{*}$ relations are shown in Fig.8; a gentle gap appears at $T \underset{f}{*}=100 \mathrm{~s}$. The bending strength oo of the 
specimens with a Vickers flaw (alumina; $98 \mathrm{~N}$, $15 \mathrm{~s}$, glass;9.8N, 30 $\mathrm{s}$ ) is shown in Fig.9 as a function of $T_{*}^{*}\left(\sigma_{0}=3 P_{\max } S /\left(2 B W^{2}\right)\right.$, alumina; $B=1$, $W=3 \mathrm{~mm}$, glass; $B=1.8, W=3 \mathrm{~mm})$. The figure shows that the $\sigma_{0}-T_{f}^{*}$ relations have a gap at the smaller value of $T_{f}$ (about $10 \mathrm{~s}$ in alumina, and $7 \mathrm{~s}$ in glass); in the specimens with a small flaw, the $\sigma_{0}-T_{f}^{*}$ (or $K_{I i}-T_{f}^{*}$ ) curve shifts to a shorter time.

In Fig.10, the $K_{T i}$-value of the glass is shown as a function of $\left(\widetilde{T}_{f}\right) \mathrm{N}$, where $a_{0}=30 \mu \mathrm{m}$ is taken as an equivalent initial crack length in the specimens with the Vickers flaw, and the experimentally determined n-values, $n_{1}=16$ and $n_{3}=40$ are used for the calculation of $\left(\widetilde{T}_{f}\right)_{N}$. Experimental data in constant load tests are also shown in the figure. The figure suggests that the $K_{I i}$-values can be expressed by a unique function of $\left(\widetilde{T}_{f}\right) N$ and the $K_{I i}-\left(\widetilde{T}_{f}\right) N$ relation consists of 4 regions.

\section{CONCLUSIONS}

By analyzing the stability condition including rate effects, it was clarified that a crack begins to extend before $P_{\max }$ appears in conventional fracture toughness tests. The amount of the extension depends on the $n$-value in the $\dot{\alpha}-\left(K_{I}\right)^{n}$ relation. A new method to analyze the failure data of the specimens with a crack was presented. Some fracture toughness and bending data in an alumina ceramic and a glass showed that a clear bend appears in the $K_{I i}-T *$ relation, and the time to failure becomes shorter as the initial flaw (crack) gets smaller. By using a newly presented paremeter $\left(\widetilde{T}_{f}\right)_{N}$, the data can be well expressed by a unique function of the parameter.

\section{References}

(1) Wiederhorn S.M., J. Am. Ceram. Soc., 50 (1967) 1137

(2) Evans A.G., J. Mater. Sci., 7(1972) 1137

(3) Gurney C. and Hunt J., Proc. Roy. Soc. London A299 (1967) 508

(4) Nojima T. and Nakai O., Proc. 37th Japan Congr. Mat. Res. (to be published in April 1994)

(5) Nojima T. and Katoh H., J. Japan Soc. Mat. Sci., 42(1993) 1331 (in Japanese)

Appendix

The compliance of single edge cracked 3 point bending specimen;

$$
\begin{aligned}
& \lambda=\left(1 / E^{\prime} B\right)\left|E^{\prime} B \lambda_{0}+2 \int_{0}^{x} x F^{2}(x) d x\right| \\
& F(x)=\left(\frac{S}{W}\right) \frac{3\left[1.99-x(1-x)\left(2.15-3.93 x+2.7 x^{2}\right)\right]}{2(1+2 x)(1-x)^{3 / 2}} \\
& \lambda_{0}=S^{3} /\left(4 E B W^{3}\right)
\end{aligned}
$$

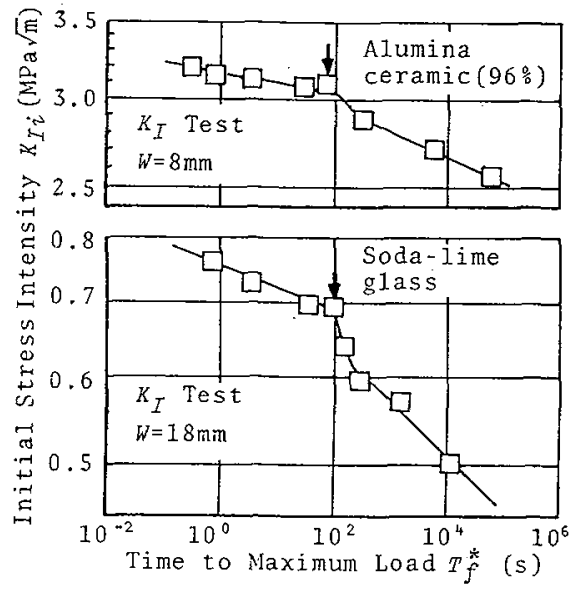

Fig. 8 Initial stress-intensity $K_{T i}-T^{*}$ relations in alumina $c e-$ ramic and soda-1ime glass
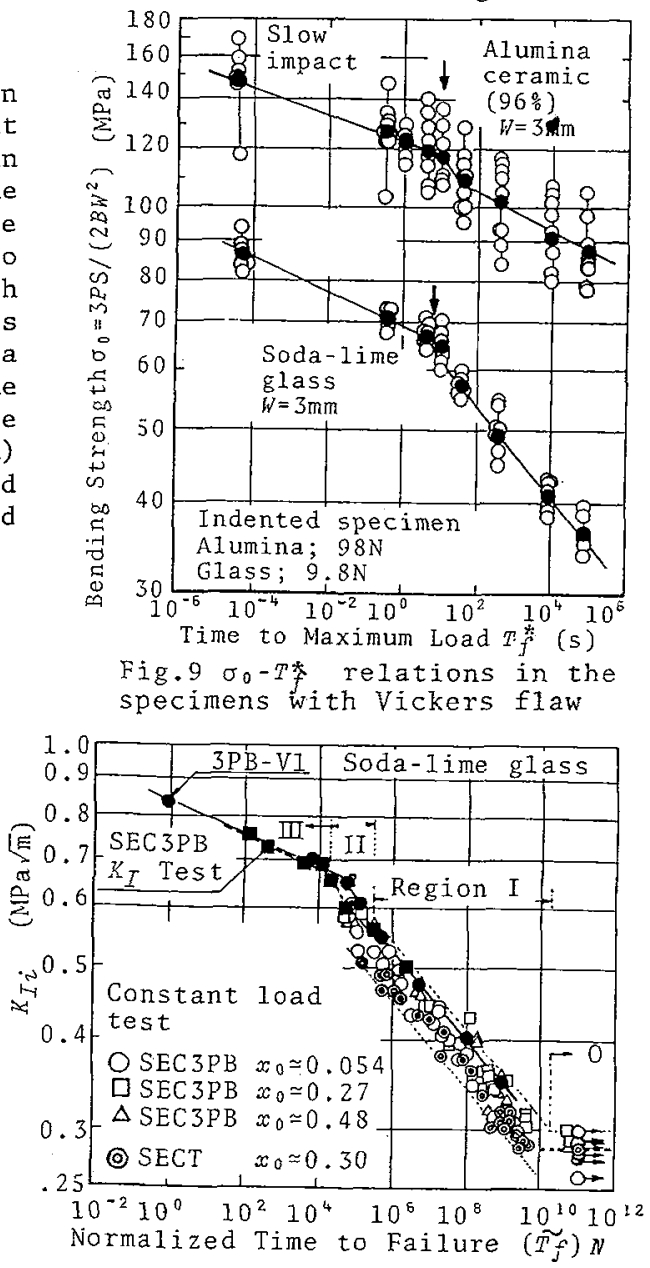

Fig. $10 K_{I i}-\left(\widetilde{T_{f}}\right)_{N}$ relations (with constant load data in $\left.\left(\widetilde{T}_{f}\right)_{N}>10^{5}\right)$ 